\title{
Coleoptera collateralia
}

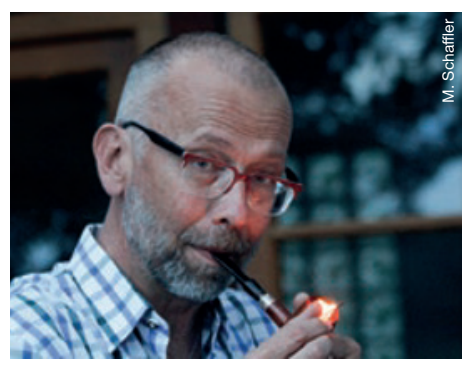

Bunte Blumen und prächtige Käfer am Weg sind gefährdet, wenn wir die Folge unbedachter Schritte nicht wägen. Schnell sind sie zertreten und alle Weisen können sie nicht mehr zusammenbauen. Es genügt ein Moment der Unachtsamkeit und das Große im Kleinen ist unwiederbringlich weg.

Ich liebe Käfer und widme dieser mit mehr als 350.000 Arten größten Ordnung des Tierreichs (so groß wie das gesamte Pflanzenreich!) seit 40 Jahren Wertschätzung, Aufmerksamkeit und großes Interesse. Das tue ich noch mehr für die weltweit verbreitete Spezies Homo sapiens.

Wenn ich mir etwas wünschen dürfte, dann mehr Aufmerksamkeit und Interesse dieser Art für sich selbst. Zu schnell sind Zusammenarbeit, Vertrauen, gemeinsame Ziele und Werte unabsichtlich und doch unachtsam zertreten und weg. Faselnde Führung ist in meinem kollateralen Weltbild eine häufig zu beobachtende Ursache dafür.

Die deutsche Kassenärztliche Bundesvereinigung (KBV) sendet dieser Tage eine klare Botschaft an die Politik: Da sich alle Bürger mittlerweile gegen COVID-19 impfen und schützen könnten, gehörten sämtliche Restriktionen aufgehoben, heißt es auf der Vertreterversammlung.

Deutschlands Vertragsärzte wollen laut Veröffentlichung der Ärztezeitung (ÄZ) raus aus der Endlosschleife der CoronaMaßnahmen. „Wenn eine Impfpflicht nicht gewollt ist - und ich will sie auch nicht -, dann gibt es politisch nur eine Alternative: Die Aufhebung aller staatlich veranlassten Restriktionen", wird der stellvertretende Vorstandschef der KBV, Stephan Hofmeister, auf der Vertretersammlung am 17.9. in Berlin vom ÄZ-Journalisten Thomas Hommel* zitiert.

Solange nicht alle Bundesbürger gegen COVID-19 geimpft werden konnten, seien einige "schwere und belastende
Einschnitte für alle" gut zu begründen gewesen, sagte Hofmeister. „Jetzt nicht mehr." Jeder, der sich mit einer Impfung schützen wolle, könne das mittlerweile problemlos tun. Daher liege es nicht mehr in der Verantwortung des Staates, sondern „in der individuellen Verantwortung jedes und jeder Einzelnen“, setzte der KBV-Vize hinzu. Der KBV-Vorstand sekundiert; ähnliche Worte fand der österreichische Bundeskanzler Anfang Juli 2021.

Die Impfung sei vor allem eine „Entscheidung für die eigene Gesundheit“, sagte Hofmeister. Das müsse die Politik auch kommunizieren. Umfragen zeigten, dass sich manche Menschen aus Protest gegen politischen Druck nicht impfen lieBen. Daher müsse „Schluss sein mit Gruselrhetorik und Panikpolitik“. Beides seien schlechte Ratgeber.

Wie humane Errungenschaften von wenigen zum Wohle vieler gepflegt werden, darüber gibt vorliegende QUALITAS - mein Dank gilt allen Autorinnen und Autoren und deren Teams - beredt Auskunft. Nur zu leicht sind sie weg, wenn man - selbstredend unabsichtlich - darauf tritt.

Nach 18 Monaten mit Schäden und Kollateralschäden im Lande darf es nicht mehr um unterschiedliche Meinungen gehen. Wenn wir etwas anstatt faselnder Führung von politischen Führungskräften erwarten dürfen, dann die Entscheidung für einen Weg, ein gemeinsames Ziel und endlich dessen konsequente Erreichung ohne die Spaltung von Menschen, Gruppen oder Gesellschaft. Mir wär's um jeden Käfer leid; noch mehr um uns.

Roland Schaffler Chefredakteur

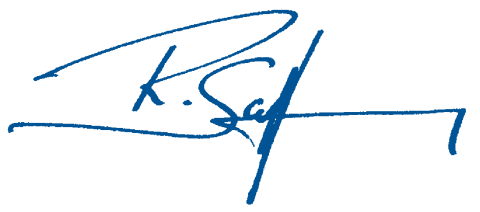

* https://www.aerztezeitung.de/Politik/ aufgerufen am 18.9.2021. 